Research article

\title{
Floristic composition and biological spectrum of weeds in agro- climatic zone of Nalbari district, Assam, India
}

\author{
D. K. Bhattacharjya ${ }^{1} *$ and S. K. Sarma ${ }^{2}$ \\ ${ }^{1}$ Corresponding author, Dept. of Botany, M.C. College, Barpeta-781301, Assam, India \\ ${ }^{2}$ Department of Botany, Gauhati University, Guwahati-781014, Assam, India \\ *Corresponding Author: dipkrbhatta@gmail.com \\ [Accepted: 02 November 2016]
}

\begin{abstract}
Present paper deals with the study of floristic composition and biological spectrum of the agro-climatic zone of Nalbari district of Assam. Field observation encompasses a total of 217 weed species from both angiosperms and pteridophytes belonging to 150 genera under 60 families. Asteraceae, being the largest family possesses 19 genera and 22 species. Out of the total species, 183 species $(84.33 \%)$ showed the annual life span as against 34 species $(15.67 \%)$ showing the perennial life span. One hundred thirty eight species $(63.59 \%)$ were recorded to be propagated by seeds/spores; 66 species (30.41\%) were found to opt for both seeds and vegetative propagules and only 13 species $(5.99 \%)$ were listed to be propagated exclusively by vegetative propagules. The phytoclimate of the agro-climatic zone can be regarded as thero-cryptophytic since major percentage of the species falls under the life forms Therophytes and Cryptophytes which has been assessed after comparison with the normal world spectrum as proposed by Raunkiaer.
\end{abstract}

Keywords: Agro-climatic zone - Weed flora - Life span - Phytodiversity - Propagation.

[Cite as: Bhattacharjya DK \& Sarma SK (2016) Floristic composition and biological spectrum of weeds in agroclimatic zone of Nalbari district, Assam, India. Tropical Plant Research 3(3): 573-585]

\section{INTRODUCTION}

Agro-climatic condition includes such basic factors of plant growth like soil types, temperature, rainfall and water availability which directly influence the vegetation of an area. Agro-climatic zone is a unit of land use in terms of major climates and the zone is favourable for certain range of crops and cultivars. Such climatic division is meant for effective and efficient management of local phytoresources, i.e. crops to meet the growing demand of food, fodder, fibre, fuel wood, timber etc. without adversely affecting the environment and causing any sort of loss to the nature (http://vikaspedia.in). India comprises a total of 15 agro-climatic zones and Assam belongs to the Eastern Himalayan zone of 15 such zones (Singh 2012).

Nalbari district of Assam is basically an agriculture dependent district. The richness of the flora of this district with a healthy combination of both primitive and advanced families is supported by the geographical position of the district and also the favourable climatic conditions. Varieties of weed species are found to infest the crop-lands, waste lands, aquatic bodies etc. in the district throughout the year. Particularly in the crop-lands, the obnoxious weeds create severe problems interfering the yield of the crops in the area. Varieties of crop cultivations are practiced throughout the year in the agro-climatic zones in Nalbari district of Assam. Along with the crops, the crop fields in the agro-climatic zones are found to be infested by a variety of weed species throughout the year.

Weeds are the plants grown in the places where they are not desired. Weeds may include all types of undesirable plants like grasses, sedges, forbs, aquatic plants, parasitic angiosperms, pteridophytic plants etc. Such plants may be the constant associates of the cultivated plants also, comprising the crop-field weeds, i.e. the vegetation infesting the human maintained crop-fields. Still the weeds have been occupying an integral part of the phytodiversity of a region.

The vegetation infesting the crop-fields of the agro-climatic zones is very often ignored and not included in the study in terms of ecology and taxonomy in comparison to forest, grassland, wetland and others. Weeds, 
although are very frequently termed as obnoxious, harmful to both human and animals and reducer of crop yield, yet a lot of weed species are useful in many ways (Bhattacharjya \& Borah 2006, Bhattacharjya et al. 2006). Apart from these, weeds have an important contribution towards the phytodiversity of a region.

Survival of mankind is directly related with the survival of phytodiversity. Being rich in phytodiversity the entire country in general and the state of Assam and entire North-East in particular possess a large number of plant species growing in a variety of climatic and edaphic conditions resulting in the formation of a wide range of habitats (Reddy et al. 2008). Floristic richness of a region enables one to have the idea about the design and functioning of the communities along with the pattern and process of the community structure (Thakur 2015). Study of floristic composition is considered as fundamental and regarded as prerequisite for all kinds of ecological research. All other works revolve around the floristic study (Naveed et al. 2012).

Climatic, edaphic and biotic factors prevailing in an area influence the formation of vegetation of that area (Shahid \& Joshi 2015). Existing vegetation is also an indicator of the climate, soil and anthropogenic influences occurring in a region (Sharma et al. 2014). The major community description and its appearance depend upon the occurrence of life forms which are based on the position and degree of protection of regenerating parts with respect to the ground surface (Cain 1950). The physical appearance of vegetation chiefly depends on the life form of dominant plant species (Hanson \& Churchill 1961). Life form pattern of the species and the proportion of life forms in an area reflect a complete ecological picture of the community as well as provide a good indication of the climatic zone of the community (Cain 1950, Kershaw 1973).

The plant species of any community can be classified in one or the other life forms. The ratio of the life forms of different species in terms of numbers or percentages in any floristic community is the biological or phytoclimatic spectrum. The biological spectrum is also regarded as the indicative of the prevailing environment as the life forms are related to the environment around the plants (Sudhakar Reddy et al. 2011).

Plant's life form is one among its most striking characteristics. In classifying vegetation, Raunkiaer's life form system is very widely used as such or with a few modifications (Braun-Blanquet 1951, Dansereau 1957a, Mueller-Dombois \& Ellenberg 1974). Analyzing the life forms of various regions of the world and comparing them with a normal spectrum based on 1,000 species selected at random, Raunkiaer reported a predominance of Phanerophytes in tropical moist regions. Following the same principle, a high preponderance of Therophytes and not insignificant proportions of Chamaephytes and Hemicryptophytes may be found from a desert area (Raunkiaer 1934).

There has been an increasing effort on the study of vegetation in connection with floristic composition, life forms and biological spectrum in different times (Gillespie 2004, Batalha \& Martins 2004, Reddy et al. 2011, Theilade et al. 2011, Saikia et al. 2012, Naveed et al. 2012, Burja et al. 2013, Aye et al. 2014, Thakur 2015, Alemu et al. 2015). However, no such study has been conducted hitherto in the Nalbari district of Assam. Accordingly, no work is reported from the agro-climatic zone of the district in this regard. The present work, therefore, aims at to study the floristic composition of the agro-climatic zone of the district along with the preparation of biological spectrum of the agro-zone.

\section{MATERIALS AND METHODS}

The study area

Nalbari districtof Assam lies between $26^{\circ} 10^{\prime} \mathrm{N}$ to $26^{\circ} 47^{\prime} \mathrm{N}$ latitude and $90^{\circ} 15^{\prime} \mathrm{E}$ to $91^{\circ} 10^{\prime} \mathrm{E}$ longitude which occupies an area of about $1009.57 \mathrm{~km}^{2}$. The area is mainly plain. The northern side of the district is bounded by the Baksa district. The southern side by the mighty Brahmaputra. The Kamrup District falls in the east and the Barpeta District in the west. The entire area of the District is situated at the plains of the Brahmaputra Valley. The tributaries of the Brahmaputra, Nona, Buradia, Pagaldia, Borolia and Tihu which are originated from the foothills of the Himalayan Range are wild in nature and have enormous contribution towards the agrarian economy of the district. The Soil condition of the district is heterogeneous one. The Soil of the northern part is clayey and loamy, whereas middle part is loamy and sandy. The southern part is characterized by sandy soil (Industrial Profile of Nalbari district, ministry of MSME, Govt. of India). The soil $\mathrm{pH}$ varies from 5.05 to 7.22. The District has a sub-tropical climate with semi dry hot summer and cold winter. During summer, generally during the months from May to August, heavy rainfall occurs for which the district experiences flood. The District experiences annual (average) rainfall of $1500 \mathrm{~mm}$ and its humidity hovers around $80 \%$. The average temperature during summer and winter are $27.00^{\circ} \mathrm{C}$ and $16.21^{\circ} \mathrm{C}$. respectively (Office of the Deputy Commissioner, Nalbari, Assam, India). Several crop cultivations belonging to both www.tropicalplantresearch.com 
summer and winter seasons are practiced throughout the year in the district. The major crops include rice, wheat, lentil, pea, mustard, jute, sugarcane, chillis, onion, turmeric, vegetable yielding species etc.

Data collection

Frequent field visits were made for a period of three years from 2013 to 2015 to collect the weed species infesting the crop-fields under the agro-climatic zone of Nalbari district of Assam. Cultivated crop species were not taken into consideration as the species are fully human maintained and not part of natural vegetation.

Method(s) of propagation and the position of the perennating buds of each weed species were recorded on the spot by thorough observation. Life spans of the species were recorded after close observation of the life cycle right from seedling stage till death. For determination of the life-forms and analysis of the biological spectrum, Raunkiaer's system as modified by Braun-Blanquet (1951) has been followed. The percentage of each life form was calculated by using the following formula:

$$
\% \text { Life form }=\frac{\text { Number of species in any life form }}{\text { Total number of species of all life forms }} \times 100
$$

The data recorded have been presented in tabular form by arranging the families according to Bentham \& Hooker's system of classification.

\section{RESULTS}

A total of 217 weed species belonging to 150 genera and 60 families were encountered during the field study in the agro-climatic zone of Nalbari district of Assam. Normally highest number of angiospermic species (210) has been recorded from the study area in comparison to the pteridophytic species (7) (Table 1, 2; Fig. 1, 2).

Table 1. Enumeration of Angiospermic weed species and their basic ecological features. (Abbreviations: A: annual, Bl: bulbil, Ch: chamephyte, Cr: cryptophyte, Hm: hemicryptophyte, P: perennial, Of: offset, Ph: phanerophyte, R: runner, Rs: root stock, Rz: rhizome, S: seed, Sc: sucker, Sm: stem, St: stolon, Tb: tuber, Th: therophyte)

\begin{tabular}{|c|c|c|c|c|}
\hline Family & Species & Life span & $\begin{array}{c}\text { Method of } \\
\text { Propagation }\end{array}$ & Life form \\
\hline \multirow[t]{6}{*}{ Nymphaeaceae } & Euryale ferox Salisb. & $\mathrm{P}$ & $\mathrm{S}, \mathrm{Rz}$ & $\mathrm{Cr}$ \\
\hline & Nelumbo nucifera Geartn. & $\mathrm{P}$ & $\mathrm{S}, \mathrm{Rz}$ & $\mathrm{Cr}$ \\
\hline & Nymphaea alba L. & $\mathrm{P}$ & $\mathrm{Rz}, \mathrm{St}$ & $\mathrm{Cr}$ \\
\hline & N. nouchali Burm.f. & $\mathrm{P}$ & $\mathrm{Rz}, \mathrm{St}$ & $\mathrm{Cr}$ \\
\hline & N. rubra Roxb. ex Salisb. & $\mathrm{P}$ & $\mathrm{Rz}, \mathrm{St}$ & $\mathrm{Cr}$ \\
\hline & Nymphoides cristata (Roxb.) Kuntze & $\mathrm{P}$ & $\mathrm{Rz}$ & $\mathrm{Cr}$ \\
\hline Papaveraceae & Argemone mexicana $\mathrm{L}$. & A & $\mathrm{S}$ & $\mathrm{Th}$ \\
\hline \multirow[t]{2}{*}{ Brassicaceae } & Capsella bursa-pastoris (L.) Medikus. & $\mathrm{A}$ & S & $\mathrm{Ph}$ \\
\hline & Rorippa benghalensis (DC.) H. Hara. & A & S & $\mathrm{Th}$ \\
\hline Capparaceae & Cleome viscosa $\mathrm{L}$ & $\mathrm{A}$ & $S$ & $\mathrm{Th}$ \\
\hline \multirow[t]{4}{*}{ Caryophyllaceae } & Drymaria diandra Blume. & A & S & $\mathrm{Ch}$ \\
\hline & Polycarpon prostratum (Forsk.) Asch. \& Schweinf. & $\mathrm{A}$ & S & $\mathrm{Th}$ \\
\hline & Stellaria media $(\mathrm{L}$.$) Vill.$ & A & S & Th \\
\hline & S. wallichiana Haines. & A & $\mathrm{S}$ & $\mathrm{Th}$ \\
\hline Portulacaceae & Portulaca oleracea L. & A & $\mathrm{S}$ & $\mathrm{Th}$ \\
\hline Hypericaceae & Hypericum japonicum Thunb. ex Murray & A & S & $\mathrm{Th}$ \\
\hline \multirow[t]{4}{*}{ Malvaceae } & Abutilon indicum (L.) Sweet. & A & S & $\mathrm{Ph}$ \\
\hline & Malvastrum coromandalianum (L.) Garcke & $\mathrm{P}$ & $\mathrm{S}$ & $\mathrm{Ph}$ \\
\hline & Sida cordifolia $\mathrm{L}$. & $\mathrm{P}$ & S & $\mathrm{Th}$ \\
\hline & Sida rhombifolia $\mathrm{L}$. & $\mathrm{P}$ & S & $\mathrm{Th}$ \\
\hline \multirow[t]{3}{*}{ Tiliaceae } & Corchorus aestuans L. & $\mathrm{A}$ & S & $\mathrm{Ph}$ \\
\hline & Grewia sapida Roxb. & $\mathrm{P}$ & $\mathrm{S}$ & $\mathrm{Ph}$ \\
\hline & Triumfetta rhomboidea Jacq. & $\mathrm{P}$ & S & $\mathrm{Ph}$ \\
\hline Linaceae & Linum ustitatissimum L. & $\mathrm{A}$ & S & $\mathrm{Th}$ \\
\hline Balsaminaceae & Impatiens glandulifera Royle & $\mathrm{A}$ & $\mathrm{S}$ & Th \\
\hline \multirow[t]{2}{*}{ Oxalidaceae } & Oxalis corniculata $\mathrm{L}$. & A & $\mathrm{S}, \mathrm{R}$ & $\mathrm{Hm}$ \\
\hline & O. debilis H.B.K. var. corymbosa & $\mathrm{A}$ & $\mathrm{S}, \mathrm{B} 1$ & $\mathrm{Hm}$ \\
\hline Sapindaceae & Cardiospermum halicacabum L. & A & $\mathrm{S}$ & $\mathrm{Ph}$ \\
\hline \multicolumn{5}{|l|}{ Fabaceae: } \\
\hline Mimosoideae & Mimosa pudica $\mathrm{L}$. & A & S & Th \\
\hline Caesalpinioideae & Cassia sophera $\mathrm{L}$. & A & $\mathrm{S}$ & Th \\
\hline & C. tora $\mathrm{L}$. & A & $\mathrm{S}$ & $\mathrm{Ph}$ \\
\hline
\end{tabular}

www.tropicalplantresearch.com 


\begin{tabular}{|c|c|c|c|c|}
\hline \multirow[t]{9}{*}{ Papilionatae } & Aeschynomene aspera $\mathrm{L}$. & A & $\mathrm{S}$ & $\mathrm{Cr}$ \\
\hline & Aeschynomene indica $\mathrm{L}$. & A & $\mathrm{S}$ & $\mathrm{Ph}$ \\
\hline & Crotalaria juncea $\mathrm{L}$. & $\mathrm{P}$ & $\mathrm{S}$ & $\mathrm{Ph}$ \\
\hline & Desmodium gangeticum $($ L.) DC. & A & S & Th \\
\hline & D. laxiflorum DC. & A & $\mathrm{S}$ & Th \\
\hline & D. triflorum (L.) DC. & A & $\mathrm{S}$ & Th \\
\hline & D. triquetrum (L.) DC. ssp. pseudotriquetrum & A & $\mathrm{S}$ & $\mathrm{Ph}$ \\
\hline & Lathyrus aphaca $\mathrm{L}$. & A & $S$ & Th \\
\hline & Tephrosia purpurea $(\mathrm{L})$ Pers. & $\mathrm{P}$ & $\mathrm{S}$ & $\mathrm{Ph}$ \\
\hline Rosaceae & Duchesnea indica (Andrews) Focks. & A & $\mathrm{S}, \mathrm{R}$ & $\mathrm{Hm}$ \\
\hline Haloragaceae & Callitriche stagnalis Scop. & A & $\mathrm{S}$ & Th \\
\hline \multirow[t]{4}{*}{ Lythraceae } & Ammannia baccifera $\mathrm{L}$. & A & $S$ & Th \\
\hline & A. multiflora Roxb. & A & $\mathrm{S}$ & Th \\
\hline & Cuphea carthagenensis (Jacq.) J.F.Macbr. & A & S & Th \\
\hline & Rotala indica (Willd.) Cochne. & A & $\mathrm{S}$ & Th \\
\hline \multirow[t]{3}{*}{ Onagraceae } & Ludwigia adscendens (L.) Hara. & A & $\mathrm{S}$, Of & $\mathrm{Cr}$ \\
\hline & L. octavalvis (Jacq.) Raven. & A & $\mathrm{S}$ & Th \\
\hline & L. perennis L. & $\mathrm{P}$ & $\mathrm{S}$ & Th \\
\hline \multirow[t]{2}{*}{ Trapaceae } & Trapa bispinosa (Roxb.) Makino & $\mathrm{P}$ & $\mathrm{S}, \mathrm{Rz}$ & $\mathrm{Cr}$ \\
\hline & T. natans $\mathrm{L}$. & $\mathrm{P}$ & $\mathrm{S}, \mathrm{Rz}$ & $\mathrm{Cr}$ \\
\hline \multirow[t]{2}{*}{ Molluginaceae } & Glinus lotoides $\mathrm{L}$. & A & S & Th \\
\hline & Mollugo pentaphylla $\mathrm{L}$. & A & $\mathrm{S}$ & Th \\
\hline \multirow[t]{4}{*}{ Apiaceae } & Centella asiatica (L.) Urban. & A & $S, R$ & $\mathrm{Hm}$ \\
\hline & Hydrocotyle javanica thunb. & A & $S, R$ & $\mathrm{Hm}$ \\
\hline & H. sibthorpioides Lam. & A & $\mathrm{S}, \mathrm{R}$ & $\mathrm{Hm}$ \\
\hline & Oenanthe javanica (Blume) Dc. & A & $\mathrm{S}$ & Th \\
\hline \multirow[t]{3}{*}{ Rubiaceae } & Dentella repens (L.) J.R. \& G. Forst. & A & $\mathrm{S}$ & Th \\
\hline & Oldenlandia diffusa (Willd.) Roxb. & A & $S, R$ & $\mathrm{Ch}$ \\
\hline & Richardia scabra $\mathrm{L}$. & A & $\mathrm{S}, \mathrm{R}$ & Th \\
\hline \multirow[t]{22}{*}{ Asteraceae } & Blumea densiflora DC. & A & $\mathrm{S}$ & Th \\
\hline & B. lacera (Burm. f.) DC. & A & $\mathrm{S}$ & $\mathrm{Ph}$ \\
\hline & Cosmos sulfureus Cav. & A & $\mathrm{S}$ & Th \\
\hline & Cotula hemisphaerica Wall. & A & $\mathrm{S}$ & Th \\
\hline & Dichrocephala integrifolia (L.f.) O.Ktze. & A & $\mathrm{S}$ & Th \\
\hline & Eclipta prostrata $\mathrm{L}$. & A & $\mathrm{S}$ & Th \\
\hline & Elephantopus scaber $\mathrm{L}$. & A & $\mathrm{S}$ & Th \\
\hline & Enhydra fluctuans Lour. & A & $\mathrm{S}$ & $\mathrm{Cr}$ \\
\hline & Chromolaena odorata (L.) R.M.King \& H.Rob. & A & $\mathrm{S}$ & $\mathrm{Ph}$ \\
\hline & Gnaphalium luteo-album $\mathrm{L}$. & A & $\mathrm{S}$ & Th \\
\hline & G. pensylvanicum Willd. & A & $\mathrm{S}$ & Th \\
\hline & G. polycaulon Pers. & A & $\mathrm{S}$ & Th \\
\hline & Grangea maderaspatana (L.) Poiret. & A & $\mathrm{S}$ & $\mathrm{Ch}$ \\
\hline & Mikania micrantha Kunth. & A & $\mathrm{S}, \mathrm{R}$ & $\mathrm{Ph}$ \\
\hline & Parthenium hysterophorus $\mathrm{L}$. & $\mathrm{P}$ & $\mathrm{S}$ & Th \\
\hline & Sonchus wightianus DC. & A & $\mathrm{S}$ & Th \\
\hline & Sphaeranthus indicus L. & A & $\mathrm{S}$ & Th \\
\hline & Spilanthes paniculata Wallich ex DC. & A & $\mathrm{S}, \mathrm{Sc}$ & $\mathrm{Ch}$ \\
\hline & Taraxacum officinale Wigg. & A & $\mathrm{S}$ & $\mathrm{Ph}$ \\
\hline & Vernonia cinerea $($ L.) Less. & A & $\mathrm{S}$ & Th \\
\hline & Xanthium indicum Koenig. in Roxb. & A & $S$ & Th \\
\hline & Youngia japonica (L.) DC. & A & $\mathrm{S}$ & Th \\
\hline \multirow[t]{2}{*}{ Campanulaceae } & Lobelia zeylanica $\mathrm{L}$. & A & $S$ & Th \\
\hline & Wehlandbergia marginata (Thunb.) DC. & A & $\mathrm{S}$ & Th \\
\hline Hydrophyllaceae & Hydrolea zeylanica (L.) Vahl. & A & $\mathrm{S}$ & $\mathrm{Cr}$ \\
\hline \multirow[t]{2}{*}{ Boraginaceae } & Cynoglossum zeylanicum (Vahl.) Brand & A & $\mathrm{S}$ & $\mathrm{Ph}$ \\
\hline & Heliotropium indicum $\mathrm{L}$. & A & $\mathrm{S}$ & Th \\
\hline \multirow[t]{3}{*}{ Convolvulaceae } & Evolvulus mummularis $\mathrm{L}$. & A & $S, R$ & $\mathrm{Hm}$ \\
\hline & Ipomea aquatica Forst. & A & $\mathrm{S}$ & $\mathrm{Cr}$ \\
\hline & I. carnea (Mart. ex Choisy) Austin. & $\mathrm{P}$ & $\mathrm{S}, \mathrm{Sm}$. & $\mathrm{Ch}$ \\
\hline
\end{tabular}




\begin{tabular}{|c|c|c|c|c|}
\hline \multirow[t]{3}{*}{ Solanaceae } & Nicotiana plumbaginifolia Viv. & $\mathrm{A}$ & $\mathrm{S}$ & $\mathrm{Ph}$ \\
\hline & Solanum nigrum L. & A & S & Th \\
\hline & S. torvum Swartz. & A & S & $\mathrm{Ph}$ \\
\hline \multirow[t]{16}{*}{ Scrophulariaceae } & Limnophila hirsuta Benth. & A & S & Th \\
\hline & Limnophila indica (L.) Druce & A & S & $\mathrm{Cr}$ \\
\hline & L. heterophylla (Roxb.) Ben & A & S & $\mathrm{Cr}$ \\
\hline & Lindernia anagallis (Burm. f.) Pennell. & A & S & Th \\
\hline & L. antipoda (L.) Alston. & A & S & Th \\
\hline & L. ciliata (Colsm.) Pennell. & A & S & Th \\
\hline & L. cordifolia (Colsm.) Merr. & A & S & Th \\
\hline & L. crustacea (L.) Muell. & A & $S$ & Th \\
\hline & L. parviflora (Roxb.) Haines. & A & S & Th \\
\hline & L. ruelloides (Colsm.) Pennell. & A & $S$ & $\mathrm{Th}$ \\
\hline & L. tenuifolia (Colsm.) Alston. & A & S & $\mathrm{Cr}$ \\
\hline & L. viscosa (Hornem) Boldingh. & A & $\mathrm{S}$ & Th \\
\hline & Mazas pumilus (Burm.f.) Steen. & A & S & Th \\
\hline & Mecardonia procumbens (Mill.) Small & A & S & Th \\
\hline & Scoparia dulcis $\mathrm{L}$. & A & S & Th \\
\hline & Torenia diffusa D. Don. & A & $\mathrm{S}$ & $\mathrm{Th}$ \\
\hline Lentibulariaceae & Utricularia aurea Lour. & A & $\mathrm{S}, \mathrm{Of}$ & $\mathrm{Cr}$ \\
\hline \multirow[t]{4}{*}{ Acanthaceae } & Hygrophila polysperma (Roxb.) T. Anders. & A & $\mathrm{S}$ & $\mathrm{Cr}$ \\
\hline & Lepidagthis incurva D. Don. & A & S & $\mathrm{Ph}$ \\
\hline & Rostellularia japonica (Thunb.) Ellis. & A & S & Th \\
\hline & Rungia pectinata (L.) Nees. & A & S & Th \\
\hline \multirow[t]{2}{*}{ Verbenaceae } & Clerodendrum viscosum Vent. & $\mathrm{P}$ & S & $\mathrm{Ph}$ \\
\hline & Phyla nodiflora (L.) Greene. & A & S & Th \\
\hline \multirow[t]{5}{*}{ Lamiaceae } & Leonurus japonicus Houtt. & A & S & $\mathrm{Ph}$ \\
\hline & Leucas Plukenetii (Roth.) Spreng. & A & S & $\mathrm{Th}$ \\
\hline & Ocimum Basilicum L. & $\mathrm{P}$ & S & $\mathrm{Ph}$ \\
\hline & Pogostemon Fraternus Mig. & A & S & Th \\
\hline & P. Strigosus Benth. & A & S & Th \\
\hline \multirow[t]{7}{*}{ Amaranthaceae } & Achyranthes aspera $\mathrm{L}$. & A & S & Th \\
\hline & Alternanthera philoxeroides (Mart.) Griseb. & A & $\mathrm{S}$ & $\mathrm{Ch}$ \\
\hline & A. sessilis (L.) R.Br. ex DC. & A & $\mathrm{S}, \mathrm{R}$ & $\mathrm{Ch}$ \\
\hline & Amaranthus hybridus L. & A & $\mathrm{S}$ & Th \\
\hline & A. spinosus L. & A & S & $\mathrm{Th}$ \\
\hline & A. viridis $\mathrm{L}$. & A & $\mathrm{S}$ & Th \\
\hline & Celosia argentea (L.) Schinz. & A & S & Th \\
\hline Chenopodiaceae & Chenopodium album $\mathrm{L}$. & A & S & Th \\
\hline \multirow[t]{10}{*}{ Polygonaceae } & Polygonum barbatum L. & A & S & Th \\
\hline & P. glabrum Willd. & A & $\mathrm{s}$ & Th \\
\hline & $P$. chinense $\mathrm{L}$. & A & $\mathrm{S}$ & Th \\
\hline & P. hydropiper L. & A & S & Th \\
\hline & $P$. orientale L. & A & S & Th \\
\hline & P. plebeium R.Br. & A & S & Th \\
\hline & P. strigosum $\mathrm{Br}$. Prodr. & A & S & Th \\
\hline & Rumex dentatus L. & A & S & Th \\
\hline & Rumex maritimus L. & A & S & Th \\
\hline & Rumex nepalensis Spreng. & A & S & $\mathrm{Th}$ \\
\hline \multirow[t]{5}{*}{ Euphorbiaceae } & Acalypha indica L. & A & $\mathrm{S}$ & $\mathrm{Th}$ \\
\hline & Croton bonplandianum Baill. & A & $\mathrm{S}$ & Th \\
\hline & Euphorbia hirta L. & A & $\mathrm{S}, \mathrm{R}$ & $\mathrm{Ch}$ \\
\hline & E. thymifolia $\mathrm{L}$. & A & $\mathrm{S}$ & $\mathrm{Th}$ \\
\hline & Phyllanthus fraternus Webster. & A & S & $\mathrm{Th}$ \\
\hline Urticaceae & Pouzolzia zeylanica (L.) Bennett. & $\mathrm{A}$ & S & Th \\
\hline Cannabinaceae & Cannabis sativa $\mathrm{L}$. & A & $\mathrm{S}$ & $\mathrm{Th}$ \\
\hline Ceratophyllaceae & Ceratophyllum demersum L. & $\mathrm{A}$ & S, Rs & $\mathrm{Cr}$ \\
\hline \multirow[t]{3}{*}{ Hydrocharitaceae } & Hydrilla verticillata (L.f.) Royle. & $\mathrm{A}$ & $\mathrm{Rz}$ & $\mathrm{Cr}$ \\
\hline & Ottelia alismoides (L.) Pers. & A & $\mathrm{S}$ & $\mathrm{Cr}$ \\
\hline & Valisnaria spiralis L. & $\mathrm{P}$ & $\mathrm{S}, \mathrm{Rz}$ & $\mathrm{Cr}$ \\
\hline
\end{tabular}




\begin{tabular}{|c|c|c|c|c|}
\hline Zingiberaceae & Alpinia allughas (Retz.) Rosc. & $\mathrm{P}$ & $\mathrm{Rz}$ & $\mathrm{Cr}$ \\
\hline \multirow[t]{3}{*}{ Pontederiaceae } & Eichhornia crassipes (Mart.) Solms. & A & Of & $\mathrm{Cr}$ \\
\hline & Monochoria hastata (L.) Solms. & A & $\mathrm{Rz}$ & $\mathrm{Cr}$ \\
\hline & M. vaginalis C.Presl. & A & $\mathrm{Rz}$ & $\mathrm{Cr}$ \\
\hline \multirow[t]{5}{*}{ Commelinaceae } & Commelina benghalensis L. & A & $\mathrm{S}, \mathrm{R}$ & $\mathrm{Ch}$ \\
\hline & C. diffusa Burm. & A & $S, R$ & $\mathrm{Ch}$ \\
\hline & Cyanotis axillaris (L.) Don. & A & $\mathrm{S}, \mathrm{R}$ & $\mathrm{Ch}$ \\
\hline & Floscopa scandens Lour. & A & $S, R$ & $\mathrm{Ch}$ \\
\hline & Murdania nudiflora (L.) Brenan. & A & S & $\mathrm{Ch}$ \\
\hline Typhaceae & Typha elephantina Roxb. & $\mathrm{P}$ & $\mathrm{S}, \mathrm{Rz}$ & $\mathrm{Cr}$ \\
\hline \multirow{4}{*}{ Araceae } & Amorphophallus campanulatus (Roxb.) Bl. & $\mathrm{P}$ & $\mathrm{Tb}$ & $\mathrm{Ch}$ \\
\hline & Colacasia esculenta (L.) Schott. & $\mathrm{P}$ & Sc, $\mathrm{Rz}$ & $\mathrm{Hm}$ \\
\hline & Lasia spinosa Thw. & $\mathrm{P}$ & $\mathrm{S}, \mathrm{St}$ & $\mathrm{Ch}$ \\
\hline & Pistia stratiotes L. & A & $\mathrm{S}, \mathrm{St}$ & $\mathrm{Cr}$ \\
\hline \multirow[t]{2}{*}{ Lemnaceae } & Lemna purpusilla Torrey & A & $\mathrm{S}$ & $\mathrm{Cr}$ \\
\hline & Spirodela polyrrhiza (L.) Schl. & A & $\mathrm{S}, \mathrm{B}$ & $\mathrm{Cr}$ \\
\hline \multirow[t]{2}{*}{ Najadaceae } & Najas indica (Willd.) Cham. & A & S & $\mathrm{Cr}$ \\
\hline & Najas minor All. & A & $\mathrm{S}$ & $\mathrm{Cr}$ \\
\hline Aponogetonaceae & Aponogeton appendiculatus $\mathrm{H}$. Brug & A & $\mathrm{S}, \mathrm{Rs}$ & $\mathrm{Cr}$ \\
\hline \multicolumn{2}{|c|}{ Potamogetonaceae Potomogeton crispus L. } & A & $\mathrm{S}, \mathrm{B}$ & $\mathrm{Cr}$ \\
\hline \multirow[t]{3}{*}{ Alismataceae } & Alisma plantago $\mathrm{L}$. & $\mathrm{P}$ & $\mathrm{S}$ & $\mathrm{Cr}$ \\
\hline & Sagittaria guayanensis H.B. \& K. & A & $\mathrm{Tb}$ & $\mathrm{Cr}$ \\
\hline & Sagittaria sagittifolia $\mathrm{L}$. & $\mathrm{P}$ & $\mathrm{Tb}$ & $\mathrm{Cr}$ \\
\hline Eriocaulaceae & Eriocaulon viride Koern. & A & S. Tb & $\mathrm{Cr}$ \\
\hline \multirow[t]{21}{*}{ Cyperaceae } & Cyperus bulbosus Vahl. & A & $\mathrm{S}, \mathrm{Tb}$ & $\mathrm{Cr}$ \\
\hline & C. corymbosus Rottb. & $\mathrm{P}$ & $\mathrm{S}, \mathrm{Tb}$ & $\mathrm{Cr}$ \\
\hline & $C$ difformis $\mathrm{L}$. & A & $\mathrm{S}, \mathrm{Tb}$ & $\mathrm{Ch}$ \\
\hline & C. halpan $\mathrm{L}$. & A & $\mathrm{S}, \mathrm{Tb}$ & $\mathrm{Ch}$ \\
\hline & C. iria $\mathrm{L}$. & A & $\mathrm{S}, \mathrm{Tb}$ & $\mathrm{Ch}$ \\
\hline & C. pilosus Vahl. & A & $\mathrm{S}, \mathrm{Tb}$ & $\mathrm{Ch}$ \\
\hline & C. pumilus L. & A & $\mathrm{S}, \mathrm{Tb}$ & $\mathrm{Ch}$ \\
\hline & C. rotundus $\mathrm{L}$. & A & $\mathrm{S}, \mathrm{Tb}$ & $\mathrm{Ch}$ \\
\hline & C. sanguinolentus Vahl. & A & $\mathrm{S}, \mathrm{Tb}$ & $\mathrm{Ch}$ \\
\hline & C. tenuispica Steud. & A & $\mathrm{S}, \mathrm{Tb}$ & $\mathrm{Ch}$ \\
\hline & Elaeocharis dulcis (Burm.f.) Henschel. & A & S & $\mathrm{Cr}$ \\
\hline & Fimbristylis aestivalis (Retz.) Vahl. & A & $\mathrm{S}, \mathrm{Tb}$ & $\mathrm{Hm}$ \\
\hline & F. dichotoma (L.) Vahl. & A & $\mathrm{S}, \mathrm{Tb}$ & $\mathrm{Ch}$ \\
\hline & F. littoralis Gaud. & A & $\mathrm{S}, \mathrm{Tb}$ & $\mathrm{Hm}$ \\
\hline & F. miliacea (L.) Vahl. & A & $\mathrm{S}, \mathrm{Tb}$ & $\mathrm{Hm}$ \\
\hline & F. tomentosa Vahl. & $\mathrm{P}$ & $\mathrm{S}, \mathrm{Tb}$ & $\mathrm{Ch}$ \\
\hline & Kyllinga monocephela Roxb. & A & $\mathrm{S}, \mathrm{Rs}$ & $\mathrm{Cr}$ \\
\hline & Schoenoplectus articulatus (L.) & $\mathrm{P}$ & $\mathrm{S}, \mathrm{Tb}$ & $\mathrm{Cr}$ \\
\hline & S. grossuss (L.f.) & $\mathrm{P}$ & $\mathrm{S}, \mathrm{Tb}$ & $\mathrm{Cr}$ \\
\hline & Scirpus articulatus L. & A & $\mathrm{S}, \mathrm{Tb}$ & $\mathrm{Hm}$ \\
\hline & S. juncoides Roxb & A & $\mathrm{S}, \mathrm{Tb}$ & $\mathrm{Hm}$ \\
\hline \multirow[t]{15}{*}{ Poaceae } & Andropogon ascinoidis C.B.Clarke. & A & S & $\mathrm{Ch}$ \\
\hline & Axonopus compressus (Sw.) Beauv. & A & $\mathrm{S}, \mathrm{R}$ & $\mathrm{Hm}$ \\
\hline & Brachiaria distachya (L.) Stapf. & A & $\mathrm{S}$ & $\mathrm{Hm}$ \\
\hline & Cynodon dactylon (L.) Pers. & A & $\mathrm{S}, \mathrm{R}$ & $\mathrm{Hm}$ \\
\hline & Dactyloctenium aegyptium (L.) P.Beauv. & A & $\mathrm{S}, \mathrm{Tb}$ & $\mathrm{Hm}$ \\
\hline & Digitaria ciliaris (Retg.) Koel. & A & S & $\mathrm{Ch}$ \\
\hline & D. sanguinalis $\mathrm{Scop}$. & A & $S$ & $\mathrm{Ch}$ \\
\hline & Echinochloa colonum Link. & A & $\mathrm{S}$ & $\mathrm{Ch}$ \\
\hline & Eleusine indica (L.) Gaertn. & A & $\mathrm{S}, \mathrm{Rs}$ & $\mathrm{Hm}$ \\
\hline & Eragrostis coarctata Stapf. & A & S & $\mathrm{Hm}$ \\
\hline & E. tenella $($ L.) P. Beauv. & A & $\mathrm{S}, \mathrm{Tb}$ & $\mathrm{Ch}$ \\
\hline & E. unioloides (Retz.) Nees ex Steud. & A & $\mathrm{S}, \mathrm{Tb}$ & $\mathrm{Hm}$ \\
\hline & E. viscosa Trin. & $\mathrm{A}$ & $\mathrm{S}, \mathrm{Tb}$ & $\mathrm{Ch}$ \\
\hline & Eriochloa procera (Retz.) C.E.Hubb & A & $\mathrm{S}, \mathrm{Rz}$ & $\mathrm{Ch}$ \\
\hline & Phragmites karka Trin. ex Steud. & A & $\mathrm{S}$ & Th \\
\hline
\end{tabular}




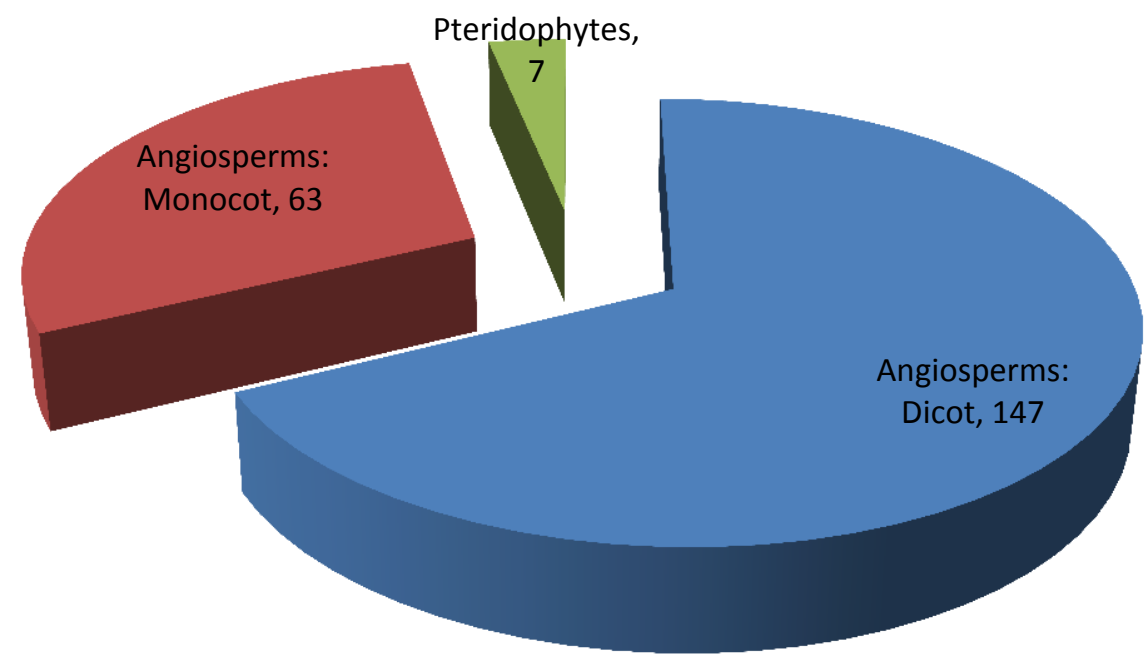

Figure 1. Plant groups and constituent number of species.

Table 2. Enumeration of Pteridophytic weed species and their basic ecological features. (Abbreviations: A: annual, Ch: chamephyte, Cr: cryptophyte, Hm: hemicryptophyte, P: perennial, R: runner, Rs: root stock, Sp: spore)

\begin{tabular}{llccc}
\hline Family & Species & Life span & $\begin{array}{c}\text { Method of } \\
\text { Propagation }\end{array}$ & Life form \\
\hline Selaginellaceae & Selaginella descipiens Warb. & $\mathrm{A}$ & $\mathrm{Sp}$ & $\mathrm{Ch}$ \\
Equisetaceae & Equisetum ramosissimum Desf. ssp. debile Hauke. & $\mathrm{A}$ & $\mathrm{Sp}, \mathrm{Rs}$ & $\mathrm{Ch}$ \\
Dryopteridaceae & Diplazium esculentum (Retz.) Swartz. & $\mathrm{P}$ & $\mathrm{Sp}, \mathrm{Rs}$ & $\mathrm{Hm}$ \\
Thelypteridaceae & Christella parasitica (L.) Holttum & $\mathrm{P}$ & $\mathrm{Sp}, \mathrm{Rs}$ & $\mathrm{Hm}$ \\
Marsiliaceae & Marsilea minuta L. & $\mathrm{A}$ & $\mathrm{Sp}, \mathrm{R}$ & $\mathrm{Cr}$ \\
& M. quadrifolia L. & $\mathrm{A}$ & $\mathrm{Sp}, \mathrm{R}$ & $\mathrm{Cr}$ \\
Azollaceae & Azolla pinnata R.Br. & $\mathrm{A}$ & $\mathrm{Sp}$ & $\mathrm{Cr}$ \\
\hline
\end{tabular}

Among the angiosperms, 147 species belonging to 104 genera and 40 families fall under dicotyledons, whereas 63 species belonging to 40 genera and 14 families undergo monocotyledons comprising a dicot-monocot ratio of 2:1, 3:1 and 3:1 for species, genera and families respectively. Among the pteridophytes, 7 species could be collected from the study area belonging to 6 genera under 6 families (Table 2). Among all the families (Angiospermic and Pteridophytic), Asteraceae (Dicot) was found to be the largest one comprising 19 genera $(12.67 \%$ ) followed by Poaceae (Monocot) comprising 11 genera (7.33\%), Fabaceae (Dicot) comprising 7 genera (4.67\%), Scrophulariaceae (Dicot) comprising 6 genera (4.00\%) etc. (Table 3). Regarding species content, the family Asteraceae (Dicot) was found to be the largest comprising 22 species (10.14\%) followed by Cyperaceae (Monocot) comprising 21 species (9.68\%), Scrophulariaceae (Dicot) comprising 16 species (7.37 $\%$ ), Poaceae (Monocot) comprising 15 species (6.91), Fabaceae (Dicot) comprising 12 species (5.53\%) etc. (Table 3). Thus the family Asteraceae has been found to possess highest number of both genera and species and can be regarded richest of all observed families in the agro-climatic zone of the district.

Table 3. Different taxa and their constituent numbers in the study area.

\begin{tabular}{|c|c|c|c|c|c|}
\hline Sl. No. & Family & No. of genera & $\%$ & No. of species & $\%$ \\
\hline \multicolumn{6}{|c|}{ Angiosperms (Dicotyledons): } \\
\hline 1 & Nymphaeaceae & 4 & 2.67 & 6 & 2.76 \\
\hline 2 & Papaveraceae & 1 & 0.67 & 1 & 0.46 \\
\hline 3 & Brassicaceae & 2 & 1.33 & 2 & 0.92 \\
\hline 4 & Capparaceae & 1 & 0.67 & 1 & 0.46 \\
\hline 5 & Caryophyllaceae & 3 & 2.00 & 4 & 1.84 \\
\hline 6 & Portulacaceae & 1 & 0.67 & 1 & 0.46 \\
\hline 7 & Hypericaceae & 1 & 0.67 & 1 & 0.46 \\
\hline 8 & Malvaceae & 3 & 2.00 & 4 & 1.84 \\
\hline 9 & Tiliaceae & 3 & 2.00 & 3 & 1.38 \\
\hline 10 & Linaceae & 1 & 0.67 & 1 & 0.46 \\
\hline 11 & Balsaminaceae & 1 & 0.67 & 1 & 0.46 \\
\hline 12 & Oxalidaceae & 1 & 0.67 & 2 & 0.92 \\
\hline 13 & Sapindaceae & 1 & 0.67 & 1 & 0.46 \\
\hline
\end{tabular}

www.tropicalplantresearch.com 


\begin{tabular}{|c|c|c|c|c|c|}
\hline \multirow[t]{4}{*}{14} & \multicolumn{5}{|l|}{ Fabaceae: } \\
\hline & Mimosoideae & 1 & & 1 & \\
\hline & Caesalpinioideae & 1 & & 2 & \\
\hline & Papilionatae & 5 & 4.67 & 9 & 5.53 \\
\hline 15 & Rosaceae & 1 & 0.67 & 1 & 0.46 \\
\hline 16 & Haloragaceae & 1 & 0.67 & 1 & 0.46 \\
\hline 17 & Lythraceae & 3 & 2.00 & 4 & 1.84 \\
\hline 18 & Onagraceae & 1 & 0.67 & 3 & 1.38 \\
\hline 19 & Trapaceae & 1 & 0.67 & 2 & 0.92 \\
\hline 20 & Molluginaceae & 2 & 1.33 & 2 & 0.92 \\
\hline 21 & Apiaceae & 3 & 2.00 & 4 & 1.84 \\
\hline 22 & Rubiaceae & 3 & 2.00 & 3 & 1.38 \\
\hline 23 & Asteraceae & 19 & 12.67 & 22 & 10.14 \\
\hline 24 & Campanulaceae & 2 & 1.33 & 2 & 0.92 \\
\hline 25 & Hydrophyllaceae & 1 & 0.67 & 1 & 0.46 \\
\hline 26 & Boraginaceae & 2 & 1.33 & 2 & 0.92 \\
\hline 27 & Convolvulaceae & 2 & 1.33 & 3 & 1.38 \\
\hline 28 & Solanaceae & 2 & 1.33 & 3 & 1.38 \\
\hline 29 & Scrophulariaceae & 6 & 4.00 & 16 & 7.37 \\
\hline 30 & Lentibulariaceae & 1 & 0.67 & 1 & 0.46 \\
\hline 31 & Acanthaceae & 4 & 2.62 & 4 & 1.84 \\
\hline 32 & Verbenaceae & 2 & 2.62 & 2 & 0.92 \\
\hline 33 & Lamiaceae & 4 & 2.67 & 5 & 2.30 \\
\hline 34 & Amaranthaceae & 4 & 2.67 & 7 & 3.23 \\
\hline 35 & Chenopodiaceae & 1 & 0.67 & 1 & 0.46 \\
\hline 36 & Polygonaceae & 2 & 1.33 & 10 & 4.61 \\
\hline 37 & Euphorbiaceae & 4 & 2.67 & 5 & 2.30 \\
\hline 38 & Urticaceae & 1 & 0.67 & 1 & 0.46 \\
\hline 39 & Cannabinaceae & 1 & 0.67 & 1 & 0.46 \\
\hline 40 & Ceratophyllaceae & 1 & 0.67 & 1 & 0.46 \\
\hline \multicolumn{6}{|c|}{ Angiosperms (Monocotyledons): } \\
\hline 41 & Hydrocharitaceae & 3 & 2.00 & 3 & 1.38 \\
\hline 42 & Zingiberaceae & 1 & 0.67 & 1 & 0.46 \\
\hline 43 & Pontederiaceae & 2 & 1.33 & 3 & 1.38 \\
\hline 44 & Commelinaceae & 4 & 2.67 & 5 & 2.30 \\
\hline 45 & Typhaceae & 1 & 0.67 & 1 & 0.46 \\
\hline 46 & Araceae & 4 & 2.67 & 4 & 1.84 \\
\hline 47 & Lemnaceae & 2 & 1.33 & 2 & 0.92 \\
\hline 48 & Najadaceae & 1 & 0.67 & 2 & 0.92 \\
\hline 49 & Aponogetonaceae & 1 & 0.67 & 1 & 0.46 \\
\hline 50 & Potamogetonaceae & 1 & 0.67 & 1 & 0.46 \\
\hline 51 & Alismataceae & 2 & 1.33 & 3 & 1.38 \\
\hline 52 & Eriocaulaceae & 1 & 0.67 & 1 & 0.46 \\
\hline 53 & Cyperaceae & 6 & 4.00 & 21 & 9.68 \\
\hline 54 & Poaceae & 11 & 7.33 & 15 & 6.91 \\
\hline \multicolumn{6}{|c|}{ Pteridophytes: } \\
\hline 55 & Selaginellaceae & 1 & 0.67 & 1 & 0.46 \\
\hline 56 & Equisetaceae & 1 & 0.67 & 1 & 0.46 \\
\hline 57 & Dryopteridaceae & 1 & 0.67 & 1 & 0.46 \\
\hline 58 & Thelypteridaceae & 1 & 0.67 & 1 & 0.46 \\
\hline 59 & Marsiliaceae & 1 & 0.67 & 2 & 0.92 \\
\hline 60 & Azollaceae & 1 & 0.67 & 1 & 0.46 \\
\hline Tot & & 150 & 100.00 & 217 & 100.00 \\
\hline
\end{tabular}

Regarding life span of the species, 183 species $(84.33 \%)$ were found to be annual as against $34(15.67 \%)$ perennial species (Table 1; Fig. 3). Mode of propagation reveals the predominance of seed producing species. One hundred thirty eight $(63.59 \%)$ species were found to propagate only by seeds and spores (in case of pteridophytes), 66 species $(30.41 \%)$ by both seed/spore and vegetative propagules. Only 13 species $(5.99 \%)$ were found to propagate exclusively by vegetative means (Fig. 4). The vegetative propagules were recorded to be runner, sucker, corm, tuber, offset, bulbil, rhizome, stolon etc. (Table 1). 

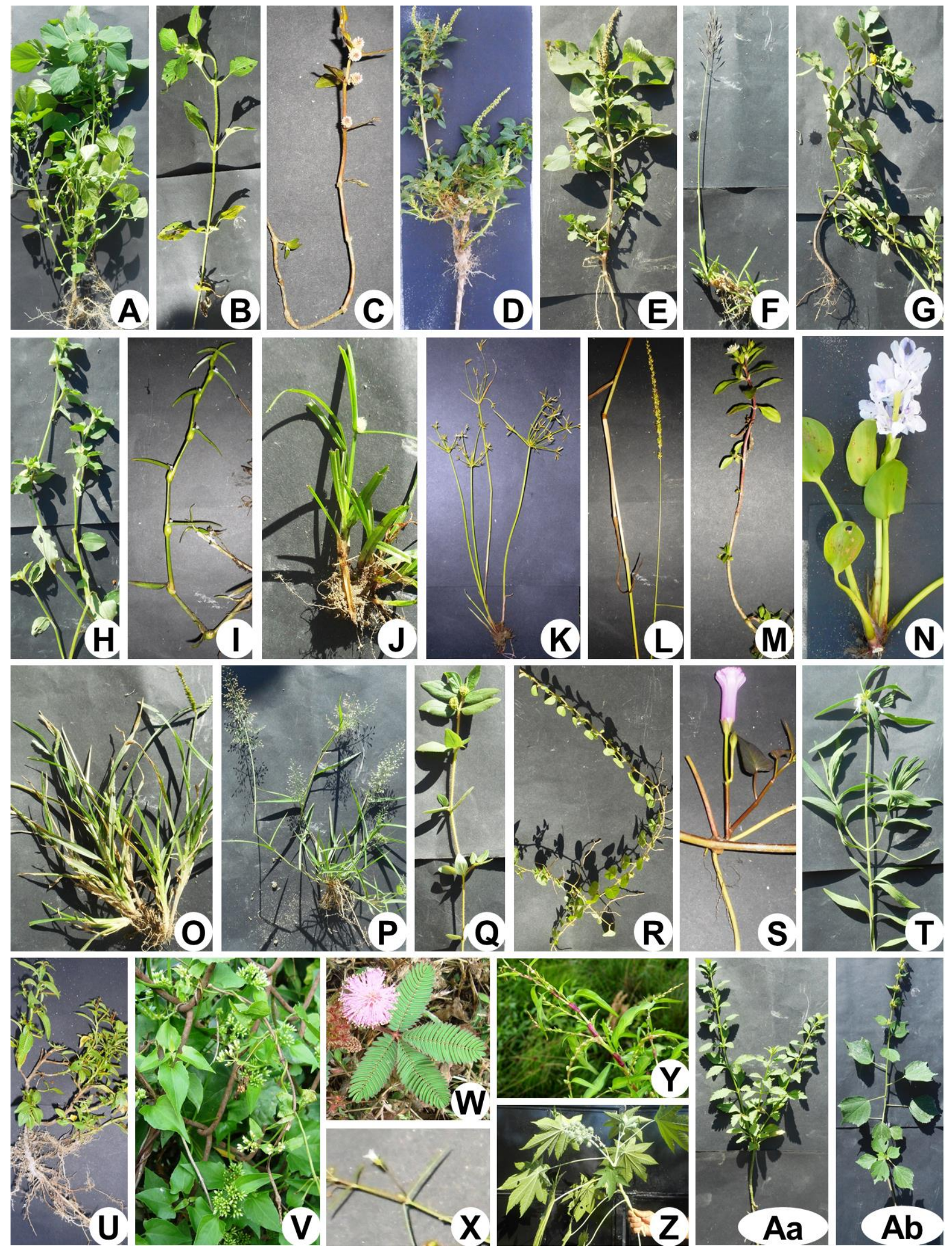

Figure 2. A, Acalypha indica; B, Ageratum conyzoides; C, Alternanthera sessilis; D, Amaranthus spinosus; E, Amaranthus viridis; F, Andropogon ascinoidis; $\mathbf{G , ~ C a s s i a ~ t o r a ; ~} \mathbf{H}$, Commelina benghalensis; I, Cyanotis axillaris; J, Cyperus bulbosus; K, Cyperus iria; L, Echinochloa colonum; M, Eclipta alba; N, Eichhornia crassipes; O, Eleusine indica; P, Eragrostis viscosa; Q, Euphorbia hirta; $\mathbf{R}$, Evolvulus nummularis; S, Ipomea aquatica; T, Leucas plukenetii; $\mathbf{U}$, Ludwigia octavalvis; V, Mikania micrantha; W, Mimosa pudica; X, Oldenlandia diffusa; Y, Persicaria hydropiper; $\mathbf{Z}$, Ricinus communis; Aa, Scoperia dulcis; Ab, Urena lobata. 


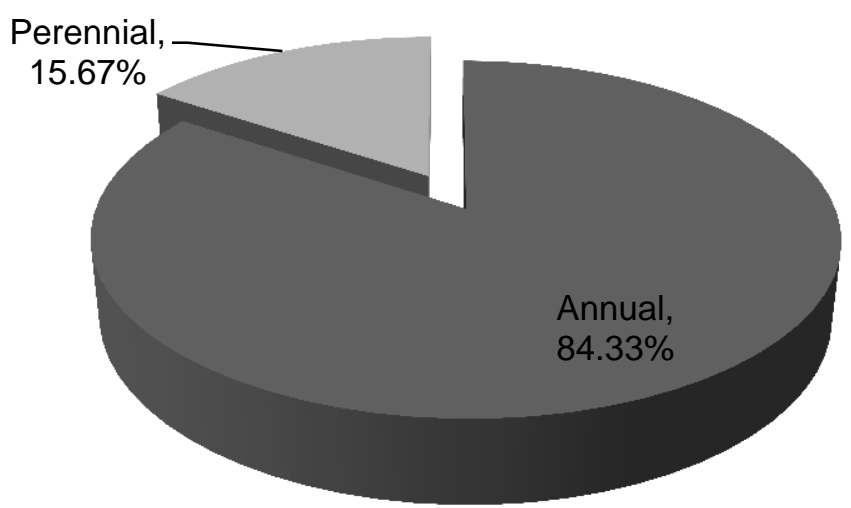

Figure 3. Life span of the species.

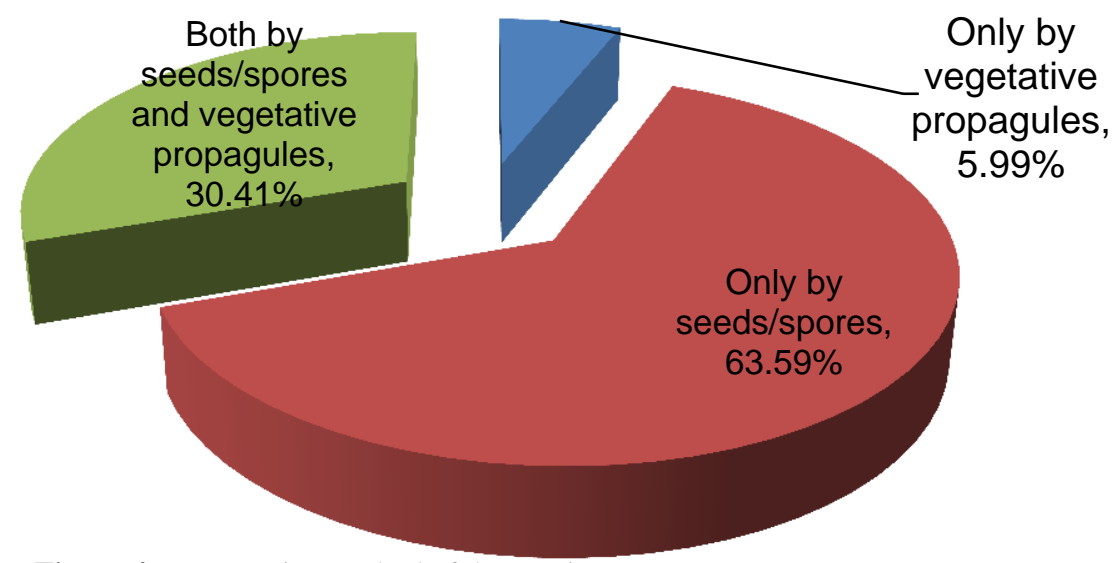

Figure 4. Propagation method of the species.

The current work was based on extensive explorations of the agro-climatic zone of the district. Out of the 217 species collected from different locations of the district, 23 species (10.60\%) belong to the life-form class Phanerophyte, 34 species $(15.67 \%)$ to Chamaephyte, 22 species $(10.14 \%)$ to Hemicryptophyte, 47 species $(21.65 \%)$ to Cryptophyte and 91 species $(41.94 \%)$ to Therophyte (Table 1,4$)$. The analysis clearly indicates the total deviation of the biological spectrum from the normal spectrum as proposed by Raunkiaer (Table 4). Study reveals the Therophytes to have the highest percentage followed by Cryptophytes and Chamephytes. On the other hand, Hemicryptophytes showed the lowest percentage. Thus, Therophytes are more abundant in the study area of the district and in contrary; the hemicryptophytes are the rare life form in the study area (Fig. 5).

Table 4. Comparative Biological spectrum.

\begin{tabular}{|c|c|c|c|}
\hline Life forms & $\begin{array}{l}\text { No. of species in } \\
\text { each life form }\end{array}$ & $\begin{array}{l}\text { Percentage distribution of the species among } \\
\text { different life forms (Observed spectrum) }\end{array}$ & $\begin{array}{c}\text { Raunkiaer's normal } \\
\text { spectrum }\end{array}$ \\
\hline Phanerophytes (Ph) & 23 & 10.60 & 46 \\
\hline Chamaephytes (Ch) & 34 & 15.67 & 9 \\
\hline Hemicryptophytes (Hm) & 22 & 10.14 & 26 \\
\hline Cryptophytes $(\mathrm{Cr})$ & 47 & 21.65 & 6 \\
\hline Therophytes (Th) & 91 & 41.94 & 13 \\
\hline Total & 217 & 100.00 & 100.00 \\
\hline
\end{tabular}

\section{DISCUSSION AND CONCLUSION}

Agro-climatic zones are rich in weed diversity. Apart from the cultivated crops, the zones are good habitat for a variety of weed species grown throughout the year (Sarma \& Bhattacharjya 2006, Bhattacharjya \& Sarma 2007, Bhattacharjya \& Sarma 2008, Padal et al. 2013, Rana \& Masoodi 2013, Dhole et al. 2013, Talukdar 2013). Present study reveals the predominance of annual weed species over the perennial ones. This is due to the anthropogenic activities including various cropping practices, weeding, collecting food and fodder species and overall control on the engineered ecosystem to gain maximum output which may reduce the growth of perennial species. Annual weeds produce very high amount of seeds to ensure propagation and survival. Sufficient amount of small seeds also ensures high probability of dispersal and re-infestation (Shivakumar et al. 2014). Thus in the 
study area, predominance of seed producing annuals is well marked. The dual method of propagation including seeds and vegetative propagules offer few weed species extra advantages to survive even in the extremes of environmental conditions.

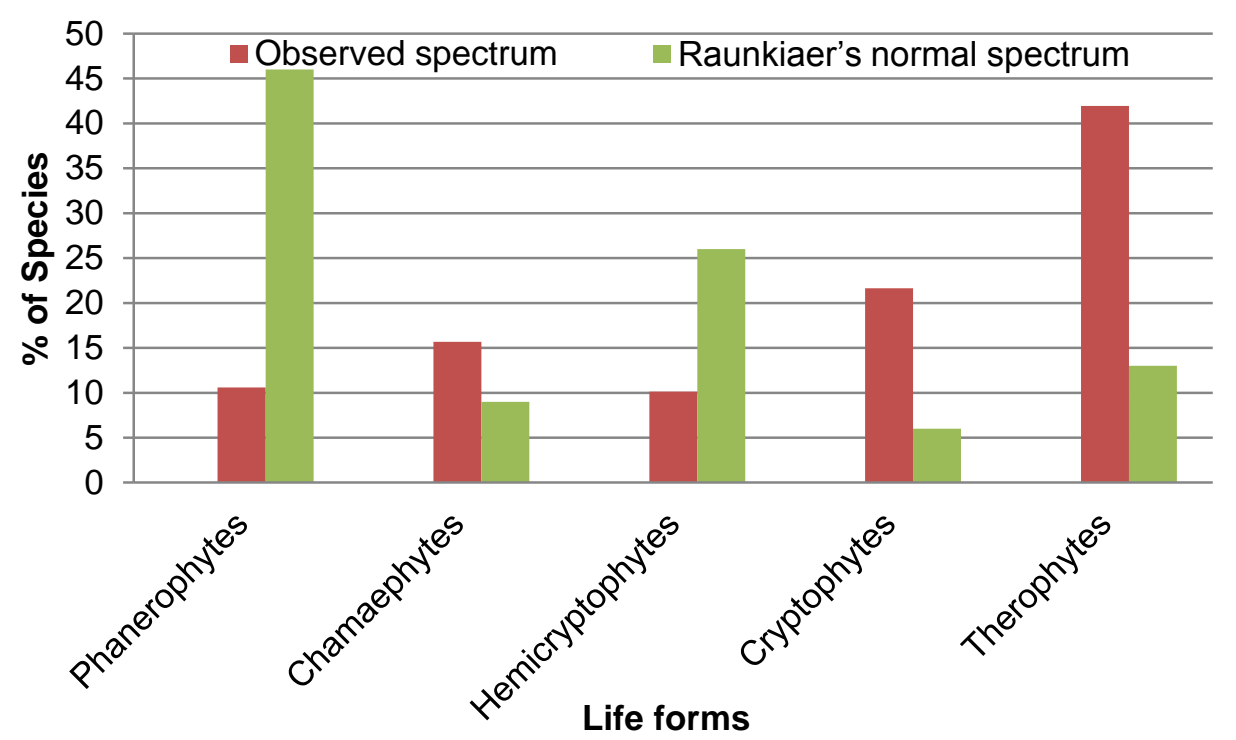

Figure 5. Comparison of observed \& normal spectrum.

The dominant life forms in biological spectrum of a region indicate the phytoclimate of that region (Yadava \& Singh 1977, Dagar \& Balakrishnan 1984, Al-Yemeni \& Sher 2010, Reddy et al. 2011, Sharma et al. 2014, Thakur 2015, Shahid \& Joshi 2015). Since the present observation indicates a higher percentage of the Therophytes, hence, the weed flora of the study area is a therophytic one which, in turn, indicates a therophytic phytoclimate prevailing in the agro-climatic zone of the district.

The rich therophytic flora is due to the grazing, weeding or other human interference in the area during the process of cultivation which reduces the number of other life forms. However the human interference has not affected the dominance of the therophytes as they produce a large number of viable seeds which, in turn, are able to establish themselves to continue their generations. Although the Hemicryptophytes are able to withstand various adverse climatic conditions along with the biotic pressure, their lower percentage value $(10.14 \%) \mathrm{kept}$ them less significant in the area. This is due to the constant human interference in the area through ploughing, hoeing, slashing, burrowing etc. associated with the agricultural practices.

It can be concluded that the vegetation of the agro-climatic zone of Nalbari district is mostly seasonal and annual weeds predominate, majority of which continue to survive in subsequent periods by their seeds or vegetative propagules and therefore their presence remains almost unchanged. Agricultural practices, grazing, scrapping by animals, collection of plants for different purposes etc. are the disturbing factors operating in the area which contribute to higher the number of therophytes and overall deviation of the biological spectrum from the normal one.

\section{ACKNOWLEDGEMENT}

Authors are thankful to Mrs. Kaberi Saikia Das, Head, Dept. of Botany, M.C. College, Barpeta (Assam) for her encouragement to conduct the work.

\section{REFERENCES}

Alemu B, Hundera K \& Abera B (2015) Floristic composition and structural analysis of Gelesha forest, Gambella regional State, Southwest Ethiopia. Journal of Ecology and the Natural Environment 7(7): 218227.

Al-Yemeni M \& Sher H (2010) Biological spectrum with some other ecological attributes of the flora and vegetation of the Asir Mountain of South West, Saudi Arabia. African Journal of Biotechnology 9(34): 5550-5559.

Aye YY, Savent P, Chanin U, Kanita T \& Nophea S (2014) Floristic Composition, Diversity and Stand Structure of Tropical Forests in Popa Mountain Park. Journal of Environmental Protection 5: 1588-1602. 
Batlaha MA \& Martins FR (2004) Floristic, frequency and vegetation life form spectra of a cerrado cite. Brazilian Journal of Biology 64 (2): 203-209.

Bhattacharjya DK \& Borah PC (2006) Importance of medicinal weeds and role of women in rural health and hygiene: a case study in Nalbari district of Assam. Indian Journal of Traditional Knowledge 7(3): 501-504.

Bhattacharjya DK \& Sarma SK (2007) Systematic Enumeration of Weeds occurring in Summer Crop-fields: a case study in Nalbari district, Assam. In: Proceeding, National Seminar on "Biodiversity Conservation- the Post-Rio Scenario in India”, Assam University, Silchar.

Bhattacharjya DK \& Sarma SK (2008) Crop Field Weeds in Nalbari District of Assam, India. Pleione 2(2): 182-189.

Bhattacharjya DK, Devi B, Sarma SK \& Das S (2006) Edible weeds in crop-fields of Nalbari district, Assam. Journal of Non-timber Forest Products 13(4): 281-286.

Braun-Blanquet J (1951) Pflanzensoziologie. Springer Verlag, Vienna.

Burju T, Hundera K \& Kelbessa E (2013) Floristic Composition and Structural Analysis of Jibat Humid Afromontane Forest, West Shewa Zone, Oromia National Regional State, Ethiopia. Ethiopian Journal of Education and Sciences 8(2): 11-33.

Cain SA (1950) Life-forms and phytoclimate. The Botanical Review 16(1): 1-32.

Dagar JC \& Balakrishnan NP (1984) Life form and biological spectrum of Andaman and Nicobar Islands. Bulletin of Botanical Survey of India 26: 154-159.

Dansereau Pierre (1957a) Biogeography: an ecological perspective. The Ronald Press Co., New York, xiii + 394 pp.

Dhole1 JA, Lone KD, Dhole, NA \& Bodke SS (2013) Studies on weed diversity of Wheat (Triticum aestivum L.) crop fields of Marathwada Region. International Journal of Current Microbiology and Applied Sciences 2(6): 293-298.

District portal of Nalbari district, Assam: Office of the Deputy Commissioner, Nalbari, Assam, India. Available from: www.nalbari.nic.in. (accessed: 16 June 2016).

Gillespie TW, Brock J \& Wright CW (2004) Prospects for quantifying structure, floristic composition and species richness of tropical forests. International Journal of Remote Sensing 25(4): 707-715.

Hanson HC \& Churchill ED (1961) The plant community. Reinhold Publishing Corp, New York. Available from: http://vikaspedia.in/agriculture/crop-production/weather-information/agro-climatic-zones-in-india (accessed: 22 May 2016).

Kershaw KA (1973) Quantitative and dynamic plant ecology (2 ${ }^{\text {nd }}$ edn.). ELBS and Edward Arnold (Publ) Ltd., London.

Mueller- Dombois, D \& Ellenberg H (1974) Aims and methods of vegetation ecology. John Wiley \& Sons, Inc. New York.

Naveed S, Hussain F, Khattak, I \& Badsha L (2012) Floristic Composition and Ecological Characteristics of Olea Acacia Forest of Shamshokii District Karak. Global Journal of Science Frontier Research Biological Science 12 (8): 30-36.

Padal SB, Sandhya Sri B, Raju Buchi \& Rama Krishna B (2013) Floristic Diversity and Indigenous Uses of Dominated Weeds in Maize Crop of Chinthapalli mandal, Visakhapatnam district, Andhra Pradesh, India. IOSR Journal of Agriculture and Veterinary Science 2(6): 56-63.

Rana D \& Masoodi H (2013) Studies on floristic diversity of an organic farm of Himachal Pradesh, India: Transformation of a barren land to a productive niche. International Journal of Biodiversity and Conservation 5(12): 810-816.

Raunkiaer C (1934) The life forms of plants and statistical plant geography. Oxford University Press, Oxford.

Reddy CS, Rao KT, Krishna ISR \& Javed SMM (2008) Vegetation and floristic studies in Nallamalais, Andhra Pradesh, India. Journal of Plant Sciences 3 (1): 85-91.

Saikia P, Choudhury BI \& Khan ML (2012) Floristic composition and plant utilization pattern in homegardens of Upper Assam, India. Tropical Ecology 53(1): 105-118.

Sarma SK \& Bhattacharjya DK (2006) Systematic study of weeds occurring in different winter crop-fields of Nalbari district of Assam. Journal of Assam Science Society 46: 27-31.

Sasaki N (2013) Floristic Composition, Diversity and Stand Structure of Tropical Forests in Popa Mountain Park. Journal of Environmental Protection 5: 1588-1602. 
Shahid M \& Joshi SP (2015) Life-forms and Biological Spectrum of Dry Deciduous Forests in Doon Valley, Uttarakhand, India.International Journal of Environmental Biology 5(1): 1-10.

Sharma J, Raina AK \& Sharma S (2014) Life form classification and biological spectrum of Lamberi Forest Range, Rajouri, J\&K, India. International Journal of Current Microbiology and Applied Sciences 3(11): 234-239.

Shivakumar KV, Devendra R, Muniswamappa MV, Halesh GK \& Mahadevamurthy M (2014) Weed seed production potentials in Bidens pilosa $\mathrm{L}$. in plantation crops in hill zone of Karnataka. International Journal of Research in Applied, Natural and Social Sciences 2 (2): 11-18.

Singh P (2012) Final report of the working group on agro-climatic zonal planning including agriculture development in north-eastern India. XI five year plan (2007-12). Volume I - main report.

Sudhakar Reddy C, Hari Krishna P, Meena SL, Ruchira Bhardwaj \& Sharma KC (2011) Composition of Life forms and Biological spectrum along climatic gradient in Rajasthan, India. International Journal of Environmental Sciences 1(7): 1632-1639.

Talukdar D (2013) Floristic Compositions and Diversity of Weed Taxa in Lentil (Lens culinnaris Medik.) Fields. Bulletin of Environment, Pharmacology and Life Sciences 2 (3): 33-39.

Thakur AS (2015) Floristic composition, life-forms and biological spectrum of tropical dry deciduous forest in Sagar District, Madhya Pradesh, India. Tropical Plant Research 2(2): 112-119.

Theilade I, Schmidt L, Chhang P \& McDonald JA (2011) Evergreen swamp forest in Cambodia: floristic composition,ecological characteristics, and conservation status. Nordic Journal of Botany 29: 71-80.

Yadava PS \& Singh JS (1977) Progress in Ecology, Vol. 2 (Grassland Vegetation). Today and Tomorrow's Print and Pub. New Delhi. 